\title{
HiRA: A Methodology for Deadlock Free Routing in Hierarchical Networks on Chip
}

\author{
Rickard Holsmark, Shashi Kumar \\ Dept. of Electronics and \\ Computer Engineering \\ Jönköping University \\ Sweden
}

\author{
Maurizio Palesi \\ Dept. of Computer Science and \\ Telecommunications Engineering \\ University of Catania \\ Italy
}

\author{
Andres Mejia \\ Dept. of Computing Engineering \\ Technical University of Valencia \\ Spain
}

\begin{abstract}
Complexity of designing large and complex NoCs can be reduced/managed by using the concept of hierarchical networks. In this paper, we propose a methodology for design of deadlock free routing algorithms for hierarchical networks, by combining routing algorithms of component subnets. Specifically, our methodology ensures reachability and deadlock freedom for the complete network if routing algorithms for subnets are deadlock free. We evaluate and compare the performance of hierarchical routing algorithms designed using our methodology with routing algorithms for corresponding flat networks. We show that hierarchical routing, combining best routing algorithm for each subnet, has a potential for providing better performance than using any single routing algorithm. This is observed for both synthetic as well as traffic from real applications. We also demonstrate, by measuring jitter in throughput, that hierarchical routing algorithms leads to smoother flow of network traffic. A router architecture that supports scalable table-based routing is briefly outlined.
\end{abstract}

Keywords: Networks on Chip, Hierarchical Networks, Deadlock Free Routing, Connectivity

\section{INTRODUCTION}

Scalability is considered to be an important, sometimes essential, property for most data communication networks. This is because the applications and usage of most networks evolve with time. The scalability property helps to reduce the time to extend the network for adding new resources and applications. Scalability property of a network is generally reflected in many aspects including topology, communication protocols, routing algorithm etc. Internet is a famous example of a scalable network.

Scalable architectures are preferred even for physically very small networks called networks-on-chip (NoC). This is because the number of cores which can be integrated on a chip is growing exponentially. For example, Intel has recently announced design and implementation of an 80-core NoC design using scalable mesh topology. Embedded system designers face the high cost of developing a complex chip. Design effort can be significantly reduced by chip architectures that can be used by many diverse applications requiring different types and number of resources. Like any large and complex system, concept of hierarchy will be useful for design, analysis, testing, usage and management of NoCs with a large number of cores.
One can apply the concept of hierarchy in two different ways in the NoC context. In the first way, it is a design tool for partitioning complex systems. Resources in a large flat network could be grouped in physical or logical regions or sub-networks (subnets). At higher level of abstraction the network could be treated as an interconnection of nodes, where each node is a subnet.

The concept of hierarchy can also be applied for building a large network by reusing and interconnecting many already designed NoCs. This is illustrated in Figure 1, where a hierarchical network $N$ is formed by interconnecting four individual networks. A very large network could even be organized in more than two levels, as exemplified by network $C$ that already contains a sub-network which becomes a subsub-network in network $N$. This view raises the abstraction level of reuse from resources (components) to subnets. In this case, networks at higher level consist of subnets as resources.

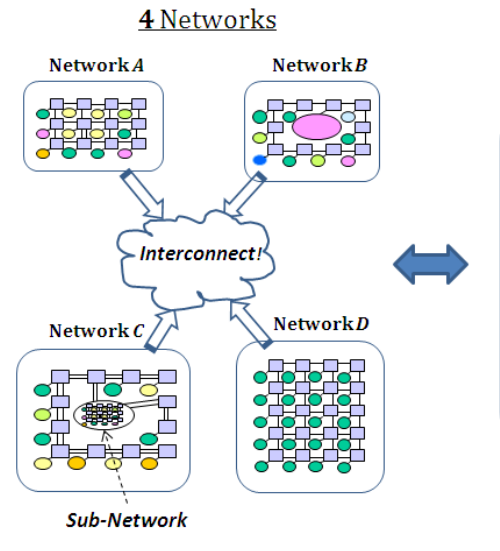

1 Network (4 Sub-Networks)

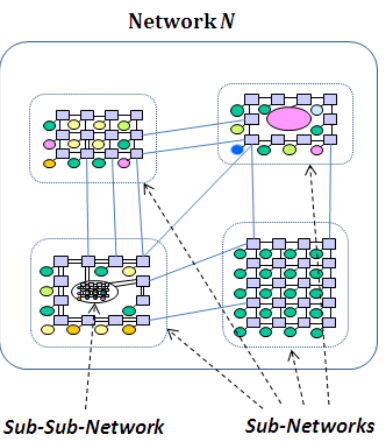

Figure 1. Hierarchical network.

Such hierarchical NoCs are likely to be heterogeneous in many different ways. Different subnets may have diverse topologies and exhibit variability in type of resources. Different subnets may also use different communication protocols and/or routing algorithms. The operating clock speeds within these subnets may also be different. Due to these dissimilarities, design and analysis of hierarchical NoCs will raise many new issues and pose many new challenges as compared to flat networks. In this paper, we deal with one important issue for large hierarchical networks, namely design of deadlock free 
routing algorithms. We call router nodes through which subnets are connected to each other as boundary nodes. A deadlock free routing methodology is described by defining rules that prevents packet deadlocks in a network of subnets.

\section{A. Scalability and Routing Algorithms}

The scalability property of a communication network is a combination of several aspects. For example, a network may have a scalable topology like mesh, but can have a non-scalable routing algorithm using a table based implementation [1] [2]. Current way to design deadlock free high performance networks, is to view all nodes and links as part of one single flat network. This view has the clear advantage that it provides the designer with the possibility to organize the network such that a certain objective function is optimized. Designing an effective routing function for such a large heterogeneous network consisting of hundreds or thousands of nodes, which guarantees deadlock freedom and which makes it possible to optimize several other objectives (e.g., routing adaptivity, link load balancing, etc.) is a computationally challenging task. As all commercial systems are constrained by business considerations; cost, quality and time to market are essential for networks in these products. In many cases, reuse of subsystems has been applied to accomplish these goals. We feel that reuse of networks will also become increasingly important.

The interesting issue of conflicting requirements for different subnets in network design can lead to a situation where a globally optimal solution is worse than a solution which is a combination of local optimal solutions for each subnet. Consider for example a network system consisting of two identifiable parts, $P 1$ and $P 2$ such that it is found that the optimal routing algorithm for the whole network is Odd-Even [3]. However, the best performance for $P 2$ individually, is achieved by using West-First [4], but the requirements of deadlock free routing restricts the number of routing algorithms to just one. If it was possible to safely mix routing algorithms in a system, higher performance could be achieved. This is the guiding motivation for our work.

\section{B. Hierarchically Designed Routing Algorithms}

To tackle the problem of poorly scalable routing algorithms, one idea could be to use a hierarchical approach as follows. Locally, each sub-network is analyzed separately from each other and the most suitable routing algorithm which allows communication between nodes of each sub-network is designed. Globally, a routing algorithm is designed for communication between the subnets. However, some consideration must be taken into account when designing the global routing algorithm. This is to avoid the situation that when subnets are put together, the resulting global routing function may not be deadlock free. The essence of designing a deadlock free routing algorithm for a network of interconnected subnets can be obtained by studying the example in Figure 2.

Figure 2 shows an interconnection of two simple subnets, where each subnet provides deadlock free internal routing by the use of bidirectional routing restrictions. But when interconnected, they are prone to deadlocks. The reason is that a cycle is formed in the channel dependency graph (CDG) of the combined network. If such cycles exist, freedom from deadlocks cannot be guaranteed [5] [6]. The cycle is caused by implicitly avoiding a routing restriction in the internal subnet using links in another subnet. It is not possible to avoid the cycle by setting restrictions on the grey "boundary nodes" that are used for connecting the subnets to each other. If such a restriction is set, the combined network is not fully connected. For example, assume a restriction that prevents the cycle by prohibiting the output of Subnet $B$ to connect to the input of Subnet $A$. Indeed, such restriction will remove all CDG cycles; but it will also remove all communication possibilities from Subnet $B$ to Subnet $A$. The only way to achieve deadlock freedom and maintaining connectivity is to modify the internal routing algorithms such that the routing restrictions are moved.

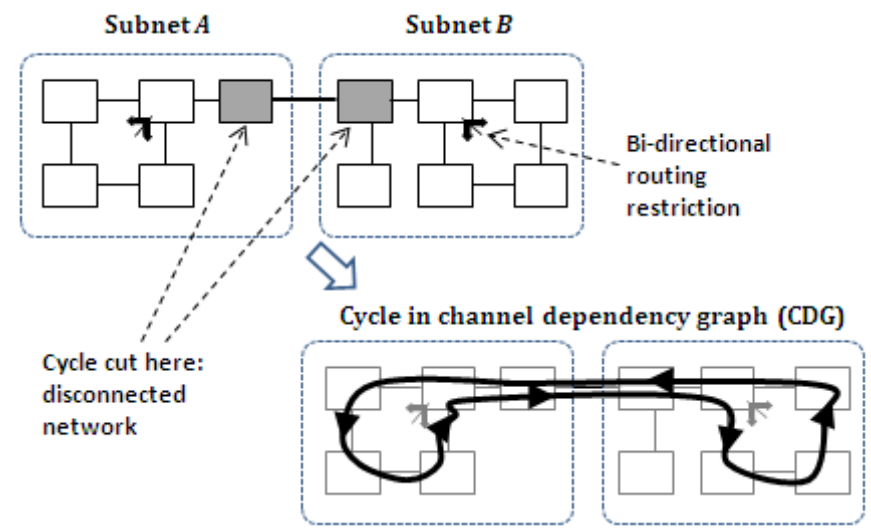

Figure 2. A deadlock-prone network with deadlock free subnets

This leads to our problem formulation of hierarchical design of deadlock free routing algorithms: Given that a large network is built by connecting many subnets where each subnet provides deadlock free routing among its internal nodes. The problem is to design a deadlock free global routing algorithm that does not affect the internal subnet routing algorithms and provides full connectivity. In this paper we propose general solutions to the deadlock issues that arise when building a network by connecting several individual deadlock free networks (subnets). We call this methodology hierarchical routing algorithms (HiRA).

\section{RELATED WORK}

The idea of hierarchy has been used to improve scalability of both on-chip and off-chip buses. Proteo NoC architecture [7] is one of the earliest hierarchical NoC proposals. The vision of Proteo was that large networks will be heterogeneous and constructed by connecting smaller subnets (referred as clusters). Clusters should be connected using bi-directional ring networks. The larger systems could be built using multiple connected rings.

Two dimensional mesh and its numerous variations have been the most popular NoC topologies. The importance of a region concept that allows irregularities in a mesh topology is discussed in [8] [9]. The stated purpose is to support varying sizes of resources, design partitioning and reuse of previously designed systems, including SoCs and NoCs. Bourduas et al. [10] have proposed a hybrid ring/mesh interconnect topology to remove limitations of large diameter of a large mesh topology 
network. In their proposal, a large mesh is partitioned into many small sub-meshes connected using hierarchical rings. They have shown that the hierarchical network topology reduces the average hop count as compared to a flat mesh network.

In [11], a hybrid mesh-ring NoC topology is proposed that is suitable for future 3D ICs. Jain et al. [12] have compared a hybrid photonic network having hierarchical mesh topology with a flat mesh network, for performance and energy consumption. A hierarchical on-chip approach is also taken in HiNoC [13], which offers both packet- and stream-based communication services. In HiNoC, the network has two levels of hierarchy; the asynchronously communicating mesh at the top level and an optional synchronously operating fat tree structure attached to a mesh router network node. Hierarchy is used in a NoC architecture based on CDMA techniques and star topology presented in [14]. Other approaches which emphasize the use of network hierarchies in the context of $\mathrm{NoC}$ based design are GigaNoC [15] and THIN [16].

Usually, hierarchical partitioning of networks refers to managing/optimization of complex network structures by splitting them into smaller sub-structures. According to our search, there is no research work reporting explicit hierarchical partitioning of a large network into non-homogeneous subnets, such that different subnets could use different routing algorithms and the whole network is still deadlock free. Although not allowing multiple routing algorithms in a network, Mejia et al. [2] use hierarchical partitioning in their SR (segment based routing) methodology.

\section{TERMINOLOGY, DeFINITIONS AND BASIC THEOREM}

The primary objective of HiRA is to provide deadlock free communication and total connectivity among network nodes of different subnets without modifying internal topologies and/or routing algorithms. Deadlock freedom is ensured by guaranteeing a cycle free channel dependency graph $(\mathrm{CDG})$ for the combined network. To achieve this, some rules regarding the interconnect of subnets must be followed.

We propose the concept of a "Safe Boundary Node" for this purpose. A node in a subnet with at least one port, used (or intended to be used) for connecting the subnet to another subnet is called a boundary node. A boundary node is safe if a deadlock cannot occur and connectivity can be guaranteed without modifying the internal routing algorithm, when it is connected to other subnet(s). We will define the properties of safe boundary nodes more formally later in the section. This section will prove that a network of interconnected deadlock free subnets will be deadlock free and connected, if the network for inter-subnet communication is deadlock free and subnets are connected to each other using only safe boundary nodes.

\section{A. General Notation and Definitions related to Deadlock Free Routing}

Variants of the definitions in this section are extensively used in literature on deadlock free routing and graph theory.

Definition 1 A network is modeled by a graph $T=(N, L)$ where each vertex $n \in N$ is a network (router-) node and each directed arc $l_{i j} \in L$, represent a unidirectional link connecting node $n_{i}$ with node $n_{j}$. Let $\operatorname{out}(n) / \operatorname{in}(n)$ represent the set of output links / input links of a node $n$ respectively, and let $\operatorname{src}(l)$ / $d s t(l)$ represent the source / destination nodes of a link $l$ respectively.

Definition 2 A path is a connected graph $P=(V, U)$, where the set of distinct vertices $V=\left\{v_{1}, v_{2}, \ldots, v_{k}\right\}$ are connected by a set of distinct edges $U=\left\{u_{12}, u_{23}, \ldots, u_{(k-1) k}\right\}$, where an edge $u_{i j}$ connects vertices $v_{i}$ and $v_{j}$. Let $p\left(v_{l}, v_{k}\right)$ indicate a path connecting and including vertex $v_{l}$ and vertex $v_{k}$. A cycle is a closed path $C=p\left(v_{l}, v_{k}\right)+u_{k l}$.

Definition 3 A routing algorithm $R$ defines all allowed message routes in a network. Each message route $p\left(n_{s}, n_{d}\right) \in R$ is a network path from a source node $n_{s}$ to a destination node $n_{d}$.

Definition 4 [5] [6] A channel dependency graph is a graph $C D G=(L, D)$ where a vertex $l \in L$, is a link in $T$ and each directed arc $d_{i j} \in D$ is a link dependency $d_{i j}=\left(l_{i}, l_{j}\right)$ such that $l_{j}$ is allowed immediately after $l_{i}$ by a routing algorithm $R$.

The routing algorithm $R$ is implemented by switching messages (packets) in network nodes. If a node $n$ and a link pair $\left(l_{\text {in }} \in \operatorname{in}(n), l_{\text {out }} \in\right.$ out $\left.(n)\right)$ is in some message route $p\left(n_{s}, n_{d}\right) \in R$, a packet is allowed to be switched from $l_{\text {in }}$ to $l_{\text {out }}$. A routing restriction between $l_{\text {in }}$ and $l_{\text {out }}$ indicates that a packet is not allowed to be switched in node $n$ from $l_{\text {in }}$ to $l_{\text {out }}$, and consequently there is no dependency $d_{\text {(in)(out })}=\left(l_{\text {in }}, l_{\text {out }}\right)$ in the CDG.

Note that the term routing algorithm is used in Definition 4. This is to indicate that the CDG, as used by our methodology is not limited to a specific routing function.

\section{B. Notation and Definitions regarding Hierarchical Network structure}

This section defines topological elements of a hierarchical network.

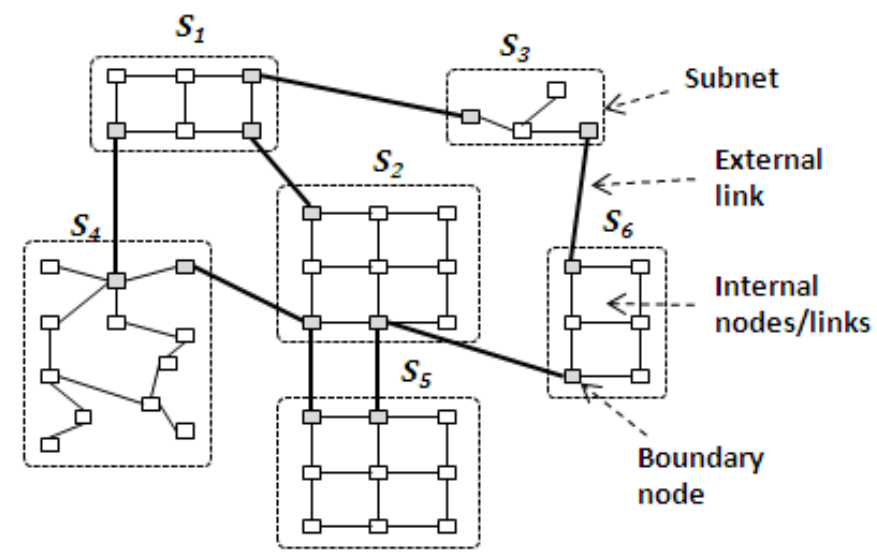

Figure 3. Hierarchical network structure.

Definition 5 A hierarchical network is a network $T_{H}=(N, L)$, partitioned into a set of disjoint subnets $S=\left\{S_{l}=\left(N_{1}, L_{1}\right), S_{2}=\left(N_{2}, L_{2}\right), \ldots, S_{k}=\left(N_{k}, L_{k}\right)\right\}$, and a set of external links $E=\{l \in T: l \notin L(\cup S)\}$. 
Definition 6 The boundary nodes in a hierarchical network interconnect subnets via external links. Formally, the set of boundary nodes are $B=\{n \in N: \exists l \in E: \operatorname{src}(l) v d s t(l)=n\}$.

As can be seen in Figure 3, all network nodes are in some subnet. Some links, however are not in any subnet. These links are the external links belonging to $E$ that connects boundary nodes of different subnet to each other. Note that all internal subnet nodes, including boundary nodes, and links of a subnet are exclusive to that subnet, since they are disjoint, i.e. $\forall S_{i}, S_{j} \in$ $S, S_{i} \cap S_{j}=\varnothing$ for $i \neq j$.

\section{Notation and Definitions on Hierarchical Deadlock Free Routing}

This section defines the components of a hierarchical routing algorithm.

Definition 7 An internal routing algorithm $R_{i}$, defines the allowed internal message routes in a subnet $S_{i}$. A message route is internal, $p\left(n_{s}, n_{d}\right) \in R_{i}$ if both the source node $n_{s}$ and the destination node $n_{d}$ are in the same subnet.

Definition 8 The external routing algorithm $R_{G}$ defines the allowed external message routes in a hierarchical network. A message route is external, $p\left(n_{s}, n_{d}\right) \in R_{G}$ if the source node $n_{s}$ and the destination $n_{d}$ are in different subnets. Any sub-path $p\left(n_{x}, n_{y}\right)$ of an external message route $p\left(n_{s}, n_{\mathrm{d}}\right)$ where all links are internal links of subnet $S_{i}$ is given by $R_{i}$.

Definition 9 The hierarchical routing algorithm $R_{H}$ defines all allowed message routes in a hierarchical network. Any message route $p\left(n_{s}, n_{d}\right) \in R_{H}$ is given, either by a subnet internal routing algorithm $R_{i}$ or the external routing algorithm $R_{G}$.

Definition 10 A boundary node $b \in B$ in a subnet $S_{i}$ is safe if there does not exist any path $p\left(l_{\text {out }}, l_{\text {in }}\right)$ from an internal output link $l_{\text {out }}$ to an internal input link $l_{\text {in }}$ in the subnet internal CDG. In other words, $b$ is safe iff $\nexists p\left(l_{\text {out }}, l_{\text {in }}\right) \subseteq C D G_{i}$ : $l_{\text {out }} \in \operatorname{out}(b) \wedge l_{\text {in }} \in \operatorname{in}(b)$

The purpose of defining a hierarchical routing algorithm $R_{H}$ by two separate routing algorithms $R_{i}$ and $R_{G}$ is to formalize the main idea of this paper. Namely, that subnets with arbitrary internal deadlock free routing algorithms can be interconnected while maintaining deadlock freedom, without modifying the internal subnet routing algorithm. Due to this, the internal routing are independent of whether a subnet is used as a subnet or a "normal" network. A key aspect of external routing algorithm $R_{G}$ is that it has no power to affect internal message routes. If such routes are necessary for reaching a destination node, they are given by the internal routing algorithm.

The consequence of the definitions of $R_{i}$ and $R_{G}$ is that $R_{G}$ only can implement routing restrictions on boundary nodes. That is, given a dependency $d_{i j}=\left(l_{i}, l_{j}\right)$ removed by a routing restriction in $R_{G}$, either $l_{i}$ or $l_{j}$ or both must be an external link. These external restrictions can result in that some external routes from/to internal nodes are not reachable from all boundary nodes.

The implementation of a hierarchical routing algorithm is yet not defined. In the context of this paper it is assumed that $R_{H}$ is the only implemented routing algorithm. $R_{i}$ and $R_{G}$ routing algorithms are then components in the design phase of $R_{H}$. Once the routes are given by each $R_{i}$ and $R_{G}$ they are no longer used, and the hierarchical network is treated as a "normal" network. However, hierarchical routing approach provides a natural way of using two-level addressing, by implementation of $R_{i}$ and $R_{G}$ instead of $R_{H}$. This is briefly discussed in section 6 .

An external message route example is shown in Figure 4. The first part of the route $p\left(n_{s}, n_{d}\right) \in R_{G}$ is the internal route $p\left(n_{s}, b_{s}\right) \in R_{i}$ from the source node $n_{s}$ to boundary node $b_{s}$. Since it is a sub-path of $p\left(n_{s}, n_{d}\right) \in R_{G}$, it is defined by $R_{i}$. In boundary node $b_{s}, R_{G}$ routes to the boundary node $b_{d}$. The internal route $p\left(b_{d}, n_{d}\right) \in R_{j}$ again, is a sub-path of the external route and is routed by the internal routing algorithm $R_{j}$ of subnet $S_{j}$.

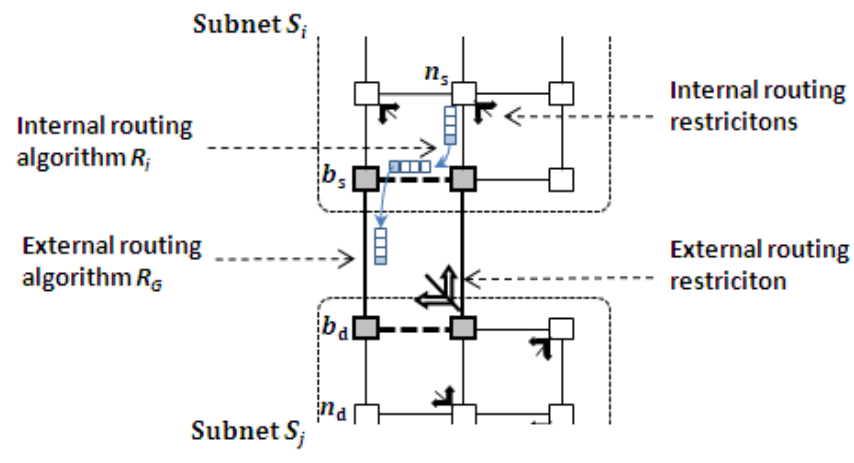

Figure 4. Internal and external routing algorithms.

A safe boundary node example is shown in Figure 5, which in upper-left corner illustrates a subnet routed by the NegativeFirst routing algorithm [4].

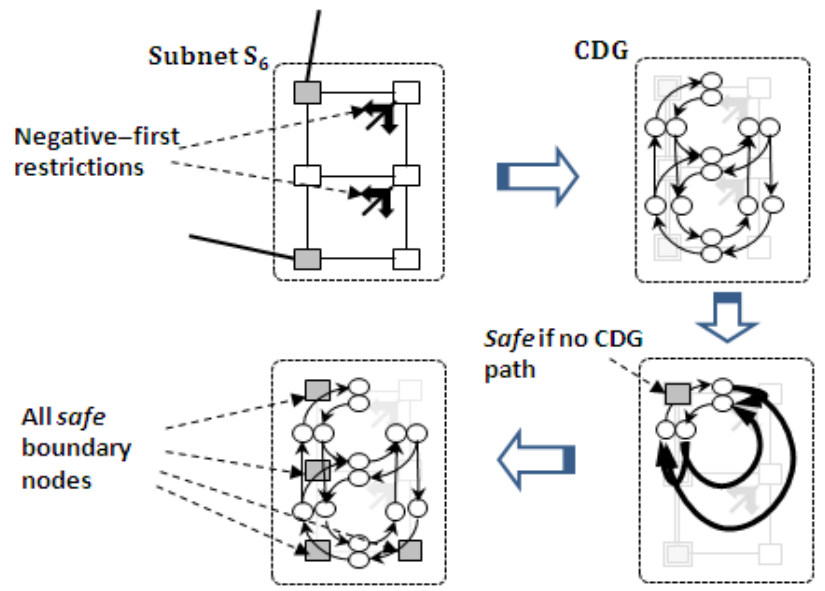

Figure 5. Safe boundary nodes.

Two grey shaded safe boundary nodes are connected to external links. To the right is the CDG of the subnet and below this is an illustration of safe node requirements of one boundary node. If it is safe, there should be no internal path formed in the CDG from any of its internal outputs to any of its internal inputs. This is shown as abstract dependencies connecting the internal links of the node. Checking all nodes for such paths reveals that there are four possible safe boundary nodes in this subnet. The other two nodes are not safe as boundary nodes. 


\section{Deadlock Freedom and Connectivity}

Now we show that a hierarchical routing algorithm is deadlock free and connected, i.e. ensures that each network node can reach any other node, for any hierarchical network configuration. Note that 1-cycles are not allowed in CDGs.

Theorem A hierarchical routing algorithm $R_{H}$ is deadlock free if

i. each subnet routing algorithm $R_{i}$ is deadlock free, and

ii. the external routing algorithm $R_{G}$ is deadlock free, and

iii. all boundary nodes are safe

\section{Proof:}

$\Leftarrow$ Consider an arbitrary subnet $S_{i}$ in a hierarchical network $T_{H}$. Internal links of this subnet $S_{i}$ may exist in $R_{i}$ and/or $R_{G}$. Let $C D G_{i}$ be acyclic by $R_{i}$, and let $C D G_{G}$ be acyclic by $R_{G}$ using routing restrictions on safe boundary nodes.

Since $R_{G}$ may define message routes that enter and leave subnets, there can exist paths in $C D G_{G}$ from external input links to external output links of boundary nodes. Therefore, in addition to restrictions on external links, $R_{G}$ may define restrictions for entering or exiting $S_{i}$ on a boundary node to avoid cycles in $C D G_{G}$. Note, as boundary nodes are safe, there cannot be a $C D G_{i}$ path from any internal output to any internal input of the same boundary node.

Assume a restriction for traversing a boundary node $b_{s}$ using an external input $l_{\text {ext_in }}$ and an internal output $l_{\text {int_out }}$. If $R_{G}$ is connected and $b_{s}$ is safe, there must be a $C D G_{G}$ path starting in an output link of the internal neighbour node of $b_{s}$, $\operatorname{dst}\left(l_{\text {int out }}\right)$, through another boundary node in $S_{i}$ to the external input $\bar{l}_{\text {ext in }}$ of $b_{s}$. If the restriction disconnects any node with $l_{\text {ext } i n}, R_{G}$ is not connected. Similarly, if there is a restriction on an internal input, $l_{\text {int } \text { in }_{\text {n }}}$ and an external output $l_{\text {ext } \text { out }}$ of a boundary node $b_{s}$, there must be a path starting in $l_{\text {ext out }}$ through $C D G_{G}$ and another boundary node in $S_{i}$ to an input link of the internal neighbour $\operatorname{src}\left(l_{\text {int in }}\right)$ in $S_{i}$. If there is a restriction on an external input $l_{\text {ext in }}$ and external output $l_{\text {ext out }}$ there must be a path from $l_{\text {ext_out }}$ to the input of the neighbour $\operatorname{src}\left(l_{\text {ext } \text { in }}\right)$ in $C D G_{G}$, if $R_{G}$ is connected.

Let $R_{H}$ be the routing algorithm that includes each subnet routing algorithm $R_{i}$ and the external routing algorithm $R_{G}$. Given that each internal $C D G_{i}$ is cycle free by $R_{i}$, an ordering can be established among the links of $C D G_{i}$ such that the order of a link $l_{j}$, in a link dependency $d_{i j}=\left(l_{i}, l_{j}\right)$ is lower than the link $l_{i}$. If there are no lower order links, $l_{j}$ is with minimum index. If $C D G_{G}$ of $R_{G}$ is cycle free, the ordering of external links and outputs/inputs of a boundary node will be constantly decreasing, where higher order links and minimal links are not necessarily in the same subnet. As there can be no dependencies other than in $C D G_{G}$ and in each subnet $C D G_{i}$, $C D G_{H}$ is cycle free.

If $R_{G}$ and each $R_{i}$ are connected, the destination node of any packet on a minimal, $l_{j}$ must always be $\operatorname{dst}\left(l_{j}\right)$. Then, $\operatorname{dst}\left(l_{j}\right)$ will sink (remove) any packet on $l_{j}$ within finite time. Let $W=\left\{w_{1}\right.$, $\left.w_{2}, \ldots, w_{k}\right\}$ be any succession of $k$ packets in $T_{H}$, where, for any two packets $\left(w_{i}, w_{i+1}\right), i=1,-2, \ldots, k-1, w_{i+1}$ is waiting on $w_{i}$. Since all packets in $W$ are routed on links in a decreasing order, $w_{h}$ in head of $W$ is either proceeding unblocked (or is on a sink) it will always advance. Then $w_{h}$ will eventually be sunk, leaving $w_{h+l}$, as new head of $W$. For each removed head $w_{h}$, each new head $w_{h+i}, i=1,-2, \ldots, k-h$ will be sunk. Then all $k$ packets in $W$ are eventually ejected from the network. As $W$ is an arbitrary succession of waiting packets, any waiting packet in any $W$ will be delivered. Thus, $R_{H}$ is connected and deadlock free.

\section{Hierarchical Routing: Design Methodologies}

This section presents basic guidelines that translate the theory in Section 3 into practical design techniques. Two scenarios are considered. In the first scenario a hierarchical network is designed using subnets that were designed without being aware that these could be part of a larger network. We call these subnets as "unaware" subnets. The other scenario is that a network is built using subnets, designed with the awareness that they will be used to build hierarchical networks. We call these subnets as "aware" subnets. In both these scenarios it is important that the subnet should have some safe boundary nodes.

\section{A. Safe Boundary Nodes}

A hierarchical deadlock free routing algorithm assumes that nodes used for interconnecting subnets are safe. The basic requirement on a safe node is that there does not exist a path of link dependencies from an output link to an input link of such node. Recall Figure 2 in Section 1 with subnets interconnected by un-safe boundary nodes. Both subnets are individually deadlock free but when interconnected, this property is lost for the full network. In this case, link dependencies combine in such a way that cycles are formed. The boundary nodes allows a path of link dependencies from its internal output links to its internal input links, which is not possible to remove by restrictions on the boundary node links, while maintaining full connectivity.

Figure 6 shows the two networks interconnected by safe boundary nodes. Both networks are deadlock free and there are no cycles in the channel dependency graph. Therefore, it is not necessary to modify the internal routing algorithms to avoid cycles that are caused only from the interconnection of the two networks. This is the main intention of hierarchical routing; interconnection of networks should not require modification of the internal routing algorithms. Restrictions on boundary node links should only be applied for communication among different subnets. In Figure 6, such restriction is not even necessary, as it should not be, since there are no possible CDG cycles formed by the external communication links.

Note that use of safe boundary nodes is a sufficient condition for hierarchical deadlock free routing. For example, it is easy to see in Figure 6 that the CDG of the interconnected subnets is cycle free even if only one of the boundary nodes is safe. Consequently, unsafe boundary nodes can be used for subnet interconnection. Still, as two unsafe nodes in many 
cases are enough to produce cyclic CDGs, the possibilities for use of unsafe nodes are limited and each configuration require careful analysis.

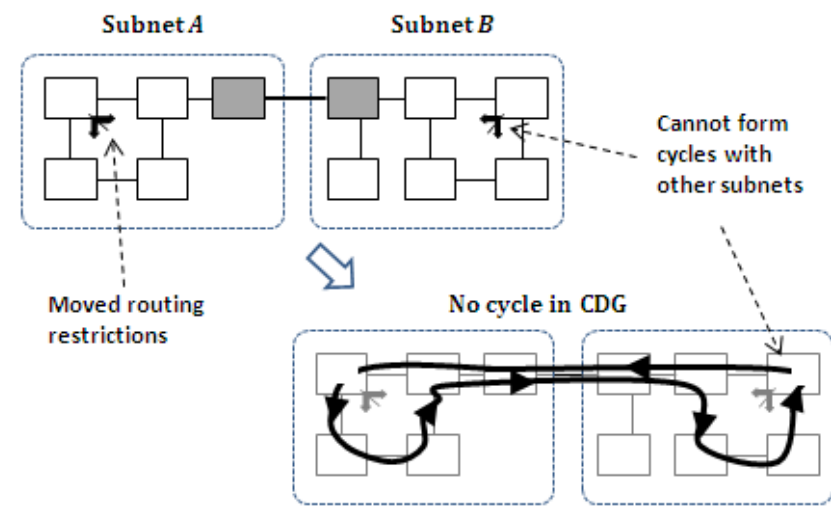

Figure 6. Deadlock free interconnection of subnets.

\section{1) Testing "safeness" of Boundary Nodes}

The safeness of a boundary node can be easily tested by using termination as shown in Figure 7 . Both the upper and lower version of the particular four-node network is deadlock free as guaranteed by an acyclic channel dependency graph, but the position of a routing restriction is different. Termination of the grey shaded boundary node creates a dependency between the external output and input links. If, considering the termination, the subnet CDG is still cycle free, the boundary node is safe. Otherwise, if cycles are found, it is not safe. As shown in Figure 7, the lower version CDG of the network is not cycle free and the boundary node is not safe.

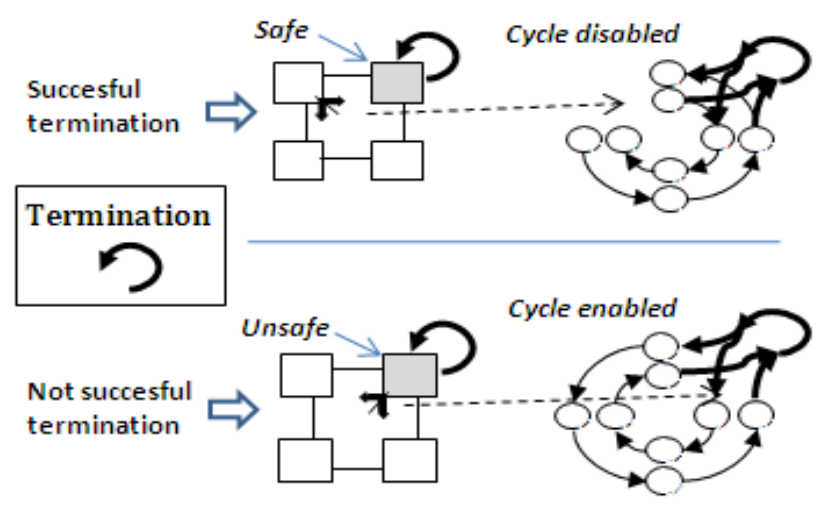

Figure 7. Termination of boundary node.

\section{2) Finding Unsafe Boundary Nodes}

In a large network consisting of hundreds of nodes, finding safe boundary nodes can be both complex and time consuming. In the following some guidelines to simplify this task is proposed.

Consider networks with deterministic routing algorithms, like for example, X-Y. Such networks allow only a single path between any source-destination pair. This automatically prevents the possibility to create a CDG path from an output link of any node to an input link of the same node. Thus, all nodes in such networks are safe boundary nodes. Next consider networks with partially adaptive routing algorithms with bidirectional routing restrictions, like for example NegativeFirst [4], Up/Down [17] and SR [2]. The possible safe boundary nodes in a network with an adaptive routing algorithm are fewer than the nodes in the network as a result of more available paths between nodes. We briefly describe an approach to quickly detect unsafe nodes in such networks.

The purpose of using safe nodes is to prevent implicitly violating an existing restriction and enabling a $\mathrm{CDG}$ cycle. Therefore a strategy to find safe nodes is to first find the routing restrictions. Consider a node $n$ with a bidirectional restriction in a network. The purpose of the restriction is to prevent one or several CDG cycles to be formed by a particular set of links. But, it is possible that a path dependency from other input to output links of $n$, not affected by the restriction, still remains even if the cycle is broken. Normally, this is not a problem since a cycle including this path will be detected and restricted at some other node. However, a safe boundary node is not allowed to reach such dependency, as it can result in a CDG cycle with another subnet. Therefore, to quickly find unsafe nodes, follow all allowed routes from the restricted input links at the restricted node $n$. The nodes visited in this path are not safe.

\section{B. A Simple Example of Combining Subnets}

The following simple example illustrates the possibilities with hierarchical routing algorithms. Consider Figure 8 and an intention to interconnect subnets with routing algorithms from left to right, Y-X, X-Y, Y-X, such that each subnet routing algorithm is kept unchanged.

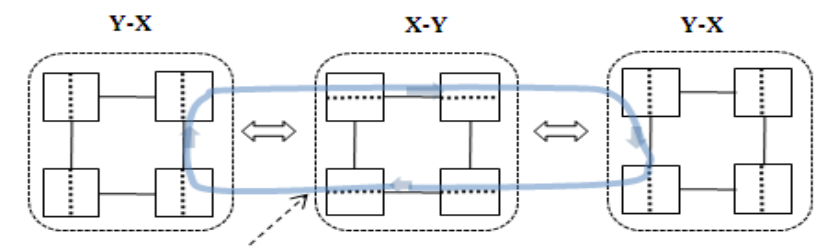

Cycle: not deadlock free

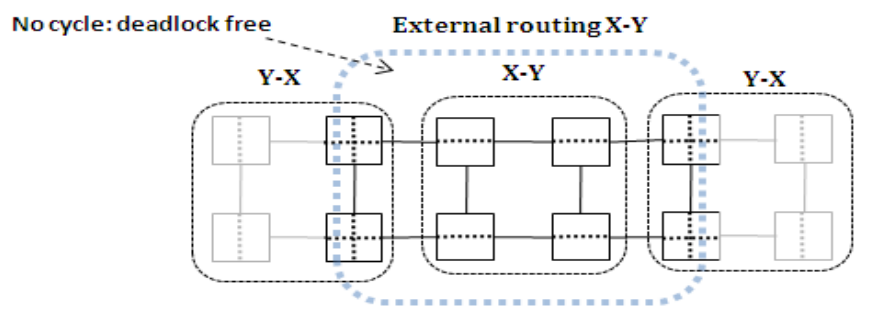

Figure 8. Interconnection of $\mathrm{X}-\mathrm{Y}$ and $\mathrm{Y}-\mathrm{X}$ networks.

Generally, creating a network routing algorithm by combining $\mathrm{Y}-\mathrm{X}$ and $\mathrm{X}-\mathrm{Y}$ routing is to beg for deadlocks. If the networks were just connected horizontally by some extra links there would be a cyclic CDG. For example, as indicated by the shaded cycle, a packet turning X-Y (west-north), waits for another taking a Y-X (north-east), which waits for a packet turning X-Y (east-south), that is blocked by a Y-X (south-west) turning packet that waits for the first packet turning $X-Y$ (west-north). A hierarchical approach could solve this by first considering the networks as subnets. Fortunately, all possible nodes are safe boundary nodes. In addition, since it is 
deterministic routing, we know that there is only one path between the boundary nodes in each subnet. Then we decide that $\mathrm{X}-\mathrm{Y}$, which in this case is the same routing algorithm as the middle subnet, is defining the external routing algorithm. This means that whenever a packet comes in and out of a subnet it is routed by X-Y. Note that even though internal subnet links are used by the external routing algorithm $\mathrm{X}-\mathrm{Y}$, the internal routing of the Y-X subnets are still Y-X.

\section{PERFORMANCE EVALUATIONS AND RESUltS}

In this section we compare a set of general purpose routing algorithms (X-Y; Negative-First, North-Last, West-First [4] and Odd-Even [3]) and some application specific routing algorithms (computed using APSRA [1]) that have been configured to see the network as a "flat network" against a hierarchical routing algorithm computed using HiRA methodology. Note that all configurations are hierarchical in the sense of global interconnection, as other routing algorithms are not usable in the described configurations. A hierarchical routing algorithm governs the entire network and the routing algorithms within the subnets, have been configured to exploit the intrinsic hierarchy of the subnets (SN). Evaluations were performed using a publically available cycle accurate NoC simulator, Noxim [18]. We consider wormhole switching with a packet size randomly distributed between 2 and 8 flits, and routers with input buffer sizes of 4 flits. We use the source packet injection rate (pir) as load parameter.

We consider four different groups of communication flows. $\mathrm{SN}->\mathrm{SN}$ refers to communication flows with source and destination located within the same subnet. SN->EN are communication flows with source node located within a SN and destination node located outside the subnet, or what is the same, in the external network (EN). The group EN->SN is communication flows with source node located outside the subnet (in EN) and destination node located within the SN in question. Finally the pass-through traffic $(\mathrm{EN}->\mathrm{SN}->\mathrm{EN})$ are communications that have both source and destination nodes located in EN but there exists at least one routing path from source to destination that passes through the $\mathrm{SN}$ in question.

\section{A. Analysis of the Impact of Global Traffic to Local Traffic}

We define as global traffic, those communication flows that involve at least two SNs (subnets). First we evaluate how global traffic affects the local traffic within a subnet. To do this, consider the network topology shown in Figure 9, which is partitioned into four subnets (A, B, C, D) interconnected through boundary nodes. We focus the analysis on the subnet labeled with A, located at the top-left corner of the topology.

This subnet has two boundary nodes located in nodes 11 and 25 . We assume that local traffic $(\mathrm{SN}->\mathrm{SN})$ within $\mathrm{A}$ has a Transpose pattern behavior with variable packet injection rate (pir). The global traffic, on the other hand, is set to a pir equal to 0.16 and consists of three kinds of communications.

- Three SN->EN communication flows in which source node is randomly selected from SN A and destination node is randomly selected from the set $\{14,49,54\}$.
- Three EN->SN communication flows where source node is randomly selected from the set $\{14,49,54\}$ and destination node is randomly selected from SN A.

- Two EN->SN->EN communication flows from node 14 to node 49 and from node 49 to node 14 .

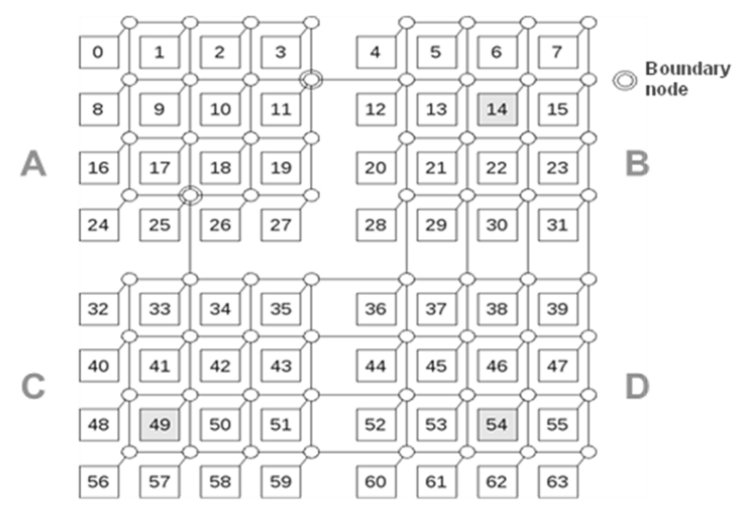

Figure 9. Network topology.

Figure 10 shows the average delay of local communications measured in A for different pir. Two different routing algorithms (X-Y and Odd-Even) have been used in A. Measurements are taken with and without global traffic.

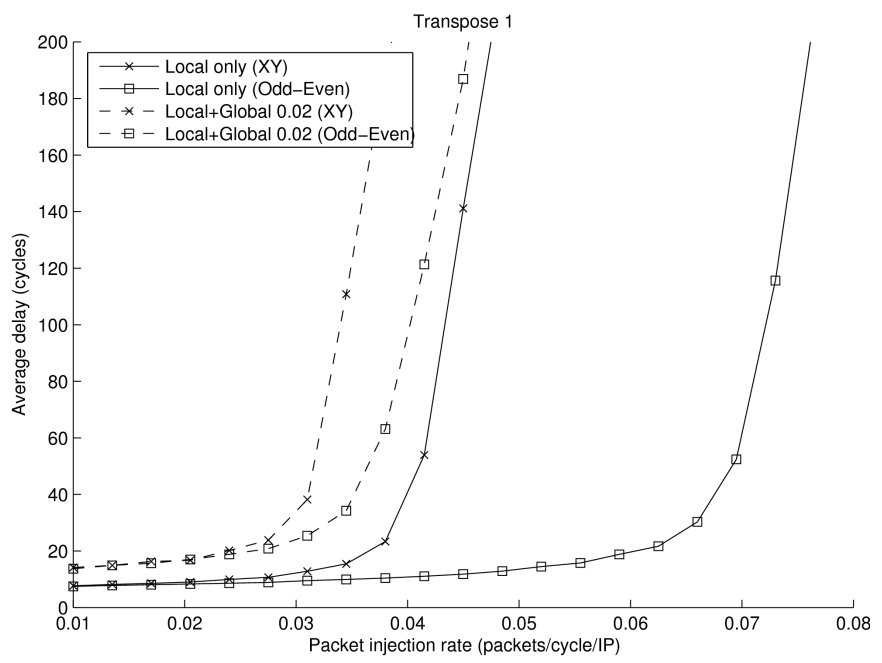

Figure 10. Average delay for local communications under transpose traffic for $\mathrm{X}-\mathrm{Y}$ and Odd-Even with and without global traffic.

As can be observed, without global traffic, the differences in performance between $\mathrm{X}-\mathrm{Y}$ and Odd-Even are very pronounced. However, in the presence of global traffic, the difference between the two algorithms reduces. This effect can be explained by observing that as soon as global traffic load increases, the local traffic deviates from pure Transpose to a mix of transpose with random traffic. As X-Y is known to perform better than Odd-Even for random traffic, this shift in traffic type has a higher impact on Odd-Even.

\section{B. Effectiveness of Hierarchical Routing}

In this experiment we consider the topology and local traffic combinations shown in Figure 11. It consists of four 
subnets (A, B, C and D) with two boundary nodes each (represented as gray tiles). Different traffic patterns are assigned to each different subnet; A uses Uniform, B presents Shuffle, C observes Transpose 1, and finally D sees a Butterfly pattern. The local pir used in each of them is such that the $\mathrm{SN}$ is below the saturation range according to the routing algorithm considered. We compare the following routing algorithms: XY, Odd-Even, West-First, and Hierarchical. The latter is a heterogeneous routing algorithm which uses $\mathrm{X}-\mathrm{Y}$ routing in subnet A, Odd-Even in subnet B, West-First in subnet $\mathrm{D}$, and Odd-Even in subnet $\mathrm{C}$. Figure 12 shows the average delay under different global traffic loads when different routing algorithms are used.

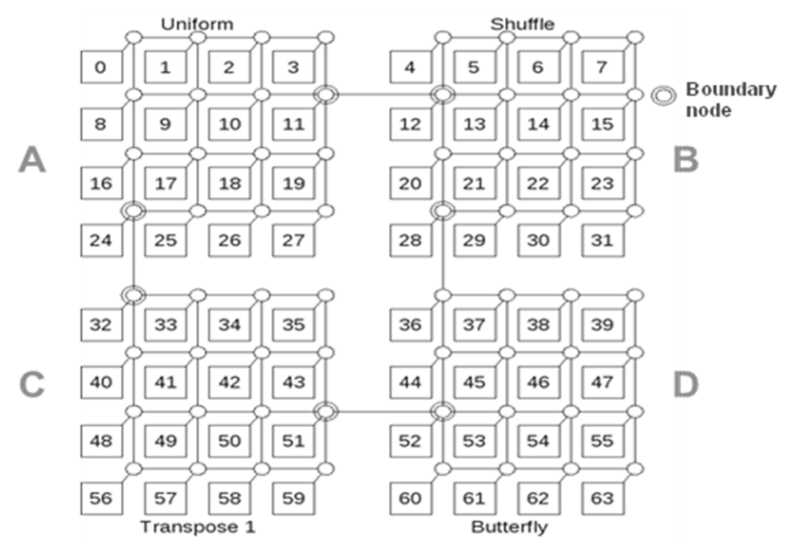

Figure 11. Network topology and traffic patterns considered in each subnet.

As a metric for the global traffic load we use the ratio between the global pir and the local pir. Measurements were taken by fixing the local traffic pir to a level below the saturation point, and varying the global traffic pir. As expected, as soon as the global to local ratio increases, overall performance of the network (measured in terms of average communication delay) decreases.

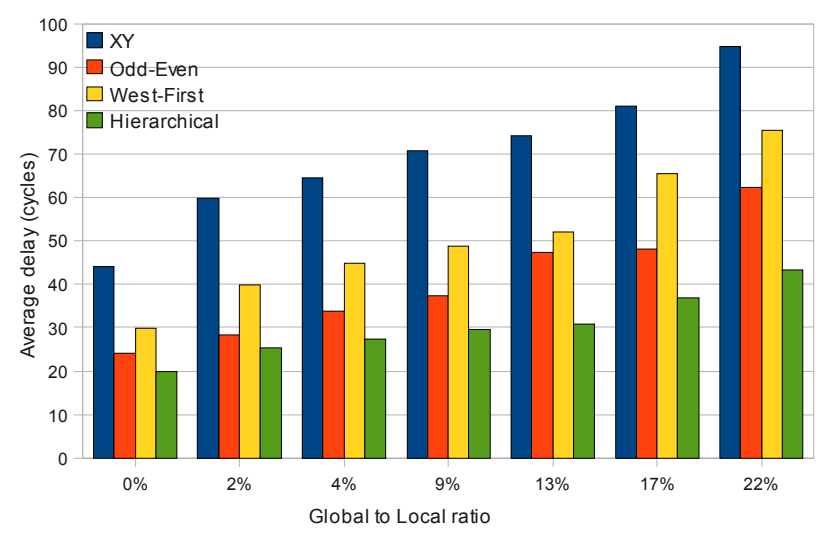

Figure 12. Average delay under different global to local ratios for different routing algorithms.

It is interesting to observe that such decrease in performance is much more pronounced in X-Y, Odd-Even, and West-First as compared to Hierarchical. This trend is analyzed in Figure 13 which shows the increase in percentage of the delay when the global to local ratio increases. As can be observed, the hierarchical routing exhibits the smallest variation.

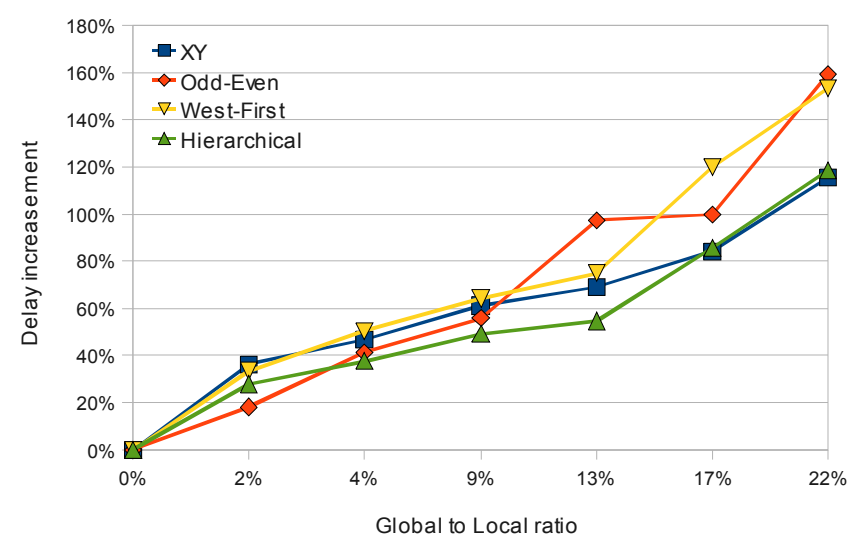

Figure 13. Increase in delay for different routing algorithms for different global to local ratios.

Finally, Figure 14 shows the percentage of performance improvement (i.e., percent average delay decrease) of hierarchical routing as compared to X-Y, Odd-Even, and WestFirst. On average, the hierarchical solution is over $50 \%, 20 \%$, and $38 \%$ more efficient (in terms of average delay) than the network configured with $\mathrm{X}-\mathrm{Y}$, Odd-Even and West-First respectively.

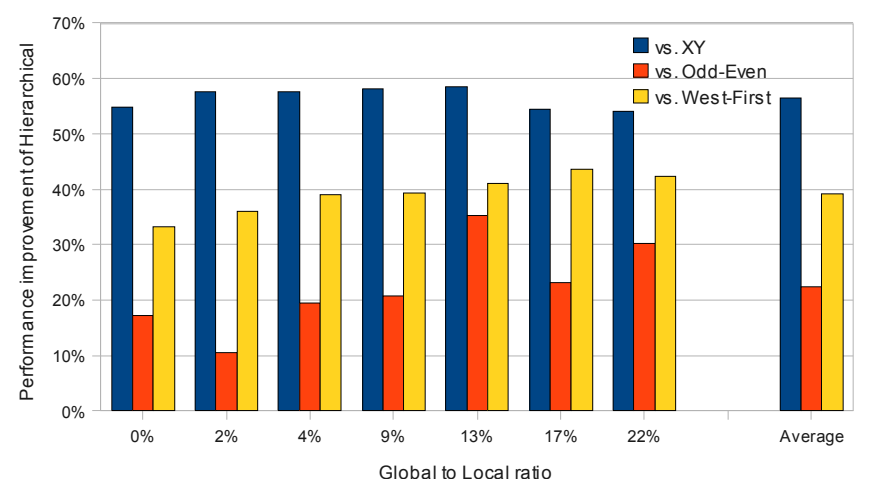

Figure 14. Percent performance improvement of hierarchical routing vs. X-Y, Odd-Even and West-First.

\section{A Real Case Study}

We also used a real case study to evaluate the benefits of hierarchical routing. Four applications were mapped on a 64 node NoC.

1. MMS: A generic MultiMedia System which includes an H.263 video encoder, an H.263 video decoder, an mp3 audio encoder and an mp3 audio decoder [19].

2. MIMO-OFDM: A MIMO-OFDM receiver in which, to support the maximum data rate of WWiSE (WorldWide Spectrum Efficiency) proposal for the nextgeneration wireless LAN systems, some of IPs have been parallelized to multiple IPs [20].

3. PIP and MWD: A Picture-In-Picture application and a Multi-Window Display application [21] [22]. 
Network topology along with the mapping of applications in shown in Figure 15. We refer to [19] [20] [21] [22] for details about the communication topology and the bandwidth requirements of the different applications. We evaluate the HiRA solution against both general purpose and application specific routing algorithms implemented for a flat network topology. For the hierarchical case, APSRA is used in the subnets where MMS-Enc and MWD are mapped, X-Y is used in subnets where MIMO-OFDM and PIP are mapped, and Negative-First is used in MMS-Dec subnet. In the other cases, the same routing algorithm is used over the entire network.

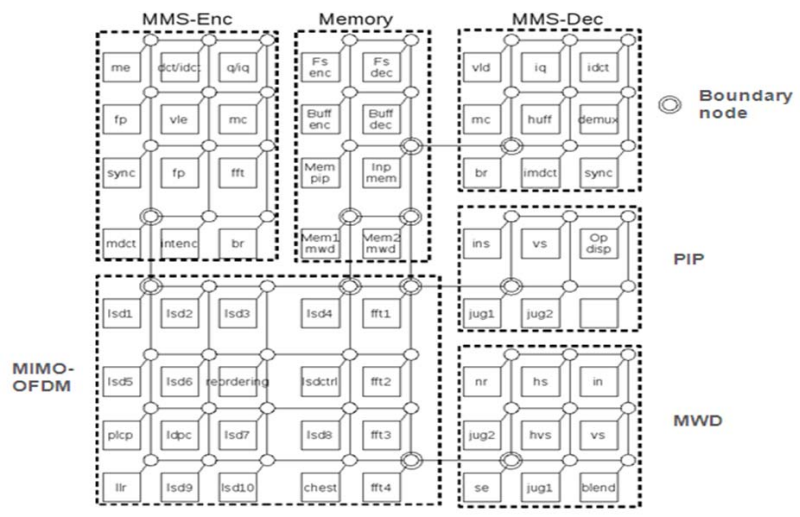

Figure 15. Network topology and mapping of applications on the NoC.

Maximum link bandwidth for the NoC is assumed to be 800 $\mathrm{MB} / \mathrm{s}$. As performance metric we use the throughput jitter which is defined as the variance in throughput during the simulation period. This parameter gives indication about how smooth the flow of traffic is in the network. Figure 16 shows the normalized throughput jitter for each subnet when a certain routing algorithm is used.

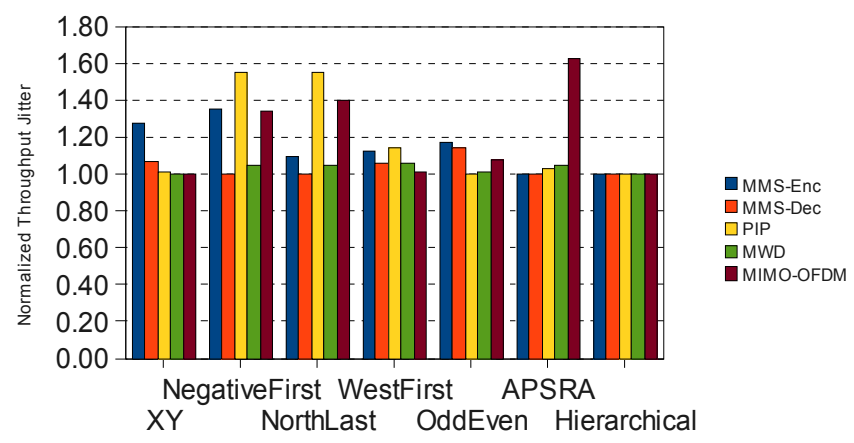

Figure 16. Normalized throughput jitter for each subnet and routing algorithm.

The values are normalized with respect to the lowest throughput jitter in that subnet. As it can be observed, hierarchical routing outperforms the other routing algorithms in each subnet. Finally, we analyze effectiveness of different routing algorithms in meeting bandwidth constraints of the applications mapped on the NoC. A communication is said to violate the link bandwidth constraints if a link used by it exceeds the maximum link bandwidth during a simulation. Figure 17 shows for each routing algorithm and for each subnet the percentage of communications that do not satisfy the link bandwidth constraints. Once again, the proposed hierarchical routing approach outperforms the other routing algorithms. On average, using hierarchical routing, only $\sim 10 \%$ of communications violate their bandwidth constraints as compared to over $\sim 20 \%$ when X-Y, Negative-First, North-Last and West-First are used and over $\sim 18 \%$ when Odd-Even and APSRA are used.

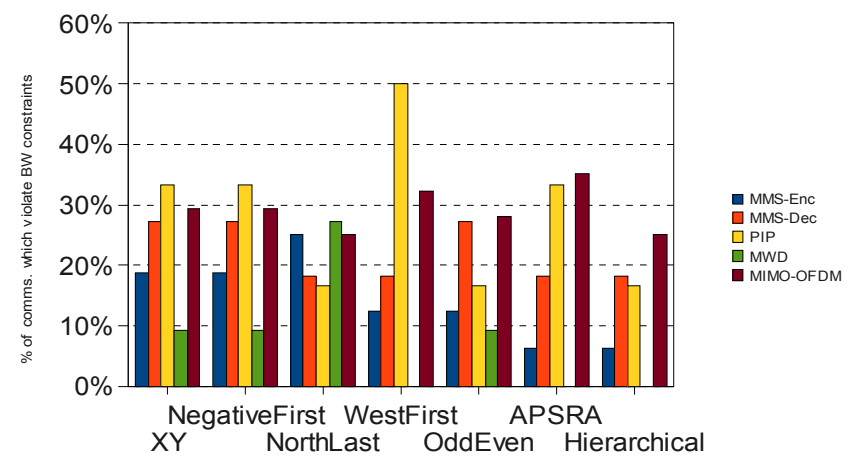

Figure 17. Percentage of communications which do not meet bandwidth constraints.

\section{SCALABLE ROUTER ARCHITECTURE}

Routing in irregular topologies, for example using Up/Down [17], is usually table-based, where sizes of the tables are dependent on the size of the network. Compression techniques [1] can reduce this problem to some extent, but in general table-based routing is not scalable.

The hierarchical deadlock free routing methodology does not imply a new router design with special features. It is possible to re-map the router address space to a flat network and use standard router design, since it is only the routes that are the important output of hierarchical deadlock-free routing. We outline logical extensions to a router design that would make routing in hierarchical networks more scalable. These ideas may also be useful for increasing scalability of other table-based routing algorithms.

Two of the most important aspects of a hierarchical network organization are the issues of addressing network nodes and implementation of routers. In this context a hierarchical approach to node addressing and routing will be logical and scalable. For example, in a two level hierarchical network the node address could be specified using two fields. The first field of the address identifies the subnet to which the node belongs and the second field specifies the position of the node within the subnet. Since a hierarchical network must be scalable, future expansion of the network must be kept in mind while deciding the size of the first field. Due to the heterogeneity of connected subnets, the size of the second field is either chosen such that it can handle the largest possible subnet, or a variable size is used dependent on the size and topology of subnet specified in the first field.

Routers for hierarchical networks pose interesting design challenges. A natural way to implement the routing function in a two-level hierarchical network is by using two tables. The first table helps in finding admissible boundary ports available for reaching the destination subnet. The second table is used for 
routing within the subnet. In both cases, if multiple routing choices are available, then the global or local network traffic information may be used for making a good path selection. It is easy to see that the first table can be by-passed for local communication. It is obvious that the hierarchical table based implementation will add some area as well as power overheads. It will also slightly slow down the computation of a routing decision. We are currently working on the design of a router for hierarchical networks that will complement HiRA methodology, with the aim to quantitatively evaluate the impact of hierarchical table based implementation on both delay, area, and power of the router.

\section{CONCLUSIONS}

Like in many other contexts, concept of hierarchy has a potential to provide many advantages but also challenges while designing, analyzing and using large and complex NoCs. Among the advantages are the possibilities for reuse of earlier designed smaller NoCs to build a large heterogeneous NoC and the possibility of virtualization of a large network as a set of many small networks for the purpose of running concurrent applications. In this paper we looked at one important issue, namely, design of deadlock free routing algorithms for hierarchical NoCs.

We described a method to design a deadlock free routing algorithm for a large network which is an interconnection of many subnets, such that each subnet has an individual deadlock free routing algorithm for internal communication among its nodes. We have modeled and evaluated hierarchical NoCs and showed that hierarchical organization has potential of providing better latency and throughput performance as compared to a large flat network for both synthetic traffic and traffic generated by a set of concurrently running real applications. We also showed that the traffic flow in a hierarchical network is smoother and has lower latency and throughput variance as compared to large flat networks. We also propose changes in a table based router design to handle hierarchical routing.

\section{REFERENCES}

[1] M. Palesi, S. Kumar, and R. Holsmark, "A Method for Router Table Compression for Application Specific Routing in Mesh Topology NoC Architectures," Proc. of the SAMOS VI Workshop: Embedded Computer Systems: Architectures, Modeling, and Simulation, 2006.

[2] A. Mejia, J. Flich, J. Duato, S.A. Reinemo, and T. Skeie, "SegmentBased Routing: An Efficient Fault-Tolerant Routing Algorithm for Meshes and Tori," Parallel and Distributed Processing Symposium, April, 2006.

[3] Ge-Ming Chiu, "The odd-even turn model for adaptive routing," Parallel and Distributed Systems, IEEE Transactions on, vol. 11, 2000, pp. 729-738.

[4] C. Glass and L. Ni, "The Turn Model for Adaptive Routing," Computer Architecture, 1992. Proceedings., The 19th Annual International Symposium on, 1992, pp. 278-287.

[5] W. Dally and C. Seitz, "Deadlock-Free Message Routing in Multiprocessor Interconnection Networks," Computers, IEEE Transactions on, vol. C-36, 1987, pp. 547-553.
[6] J. Duato, "A new theory of deadlock-free adaptive routing in wormhole networks ," Parallel and Distributed Systems, IEEE Transactions on, vol. 4, 1993, pp. 1320-1331.

[7] D. Siguenza-Tortosa and J. Nurmi, "VHDL-based simulation environment for Proteo NoC," High-Level Design Validation and Test Workshop, 2002. Seventh IEEE International, 2002, pp. 1-6.

[8] R. Holsmark and S. Kumar, "Design Issues and Performance Evaluation of Mesh NoC with Regions," NORCHIP Conference, 2005. 23rd, 2005, pp. 1-4.

[9] R. Holsmark, M. Palesi, and S. Kumar, "Deadlock Free Routing Algorithms for Mesh Topology NoC Systems with Regions," Proc. 9th EUROMICRO Conference on Digital System Design, Architectures, Methods and Tools (DSD), 2006.

[10] S. Bourduas and Z. Zilic, "A Hybrid Ring/Mesh Interconnect for Network-on-Chip Using Hierarchical Rings for Global Routing," Proc. of the ACM/IEEE Int. Symp. on Networks-on-Chip (NOCS), 2007.

[11] V. Rantala, T. Lehtonen, P. Liljeberg, and J. Plosila, "Hybrid NoC with Traffic Monitoring and Adaptive Routing for Future 3D Integrated Chips," Digest of the Workshop on Diagnostic Services in Network-onChips, 2008.

[12] A. Jain , S. Kamil, M. Mohiyuddin, J. Shalf, and J. Kubiatowicz, "Performance and Energy Comparison of Electrical and Hybrid Photonic Networks for CMPs," 2008.

[13] T. Hollstein, R. Ludewig, H. Zimmer, C. Mager, S. Hohenstern, and M Glesner, "Hinoc: A Hierarchical Generic Approach for on-Chip Communication, Testing and Debugging of SoCs," VLSI-SOC: From Systems to Chips, 2006, pp. 39-54.

[14] Daewook Kim, Manho Kim, and G. Sobelman, "CDMA-based network-on-chip architecture," Circuits and Systems, 2004. Proceedings. The 2004 IEEE Asia-Pacific Conference on, 2004, pp. 137-140 vol.1.

[15] C. Puttmann, J. Niemann, M. Porrmann, and U. Ruckert, "GigaNoC - A Hierarchical Network-on-Chip for Scalable Chip-Multiprocessors," Digital System Design Architectures, Methods and Tools, 2007. DSD 2007. 10th Euromicro Conference on, 2007, pp. 495-502.

[16] B. Qiao, F. Shi, and W. Ji, "THIN: A New Hierarchical Interconnection Network-on-Chip for SOC," Algorithms and Architectures for Parallel Processing, 2007, pp. 446-457.

[17] M. Schroeder, A. Birrell, M. Burrows, H. Murray, R. Needham, T. Rodeheffer, E. Satterthwaite, and C. Thacker, "Autonet: a high-speed, self-configuring local area network using point-to-point links," Selected Areas in Communications, IEEE Journal on, vol. 9, 1991, pp. 13181335 .

[18] F. Fazzino , M. Palesi , and D. Patti , "Noxim - NoC simulator." http://noxim.sourceforge.net/

[19] Jingcao $\mathrm{Hu}$ and R. Marculescu, "Energy- and performance-aware mapping for regular NoC architectures," Computer-Aided Design of Integrated Circuits and Systems, IEEE Transactions on, vol. 24, 2005, pp. 551-562.

[20] Sung-Rok Yoon, Jin Lee, and Sin-Chong Park, "Case Study : NoC based Next-generation WLAN receiver design in Transaction Level," Advanced Communication Technology, 2006. ICACT 2006. The 8th International Conference, 2006, pp. 1125-1128.

[21] E. Jaspers and P. de With, "Chip-set for video display of multimedia information," Consumer Electronics, IEEE Transactions on, vol. 45, 1999, pp. 706-715.

[22] E.B. van der Tol and E.G. Jaspers, "Mapping of MPEG-4 decoding on a flexible architecture platform," Media Processors 2002, San Jose, CA, USA: SPIE, 2001, pp. 1-13. 